\title{
THE EFFECT OF BODY WEIGHT AND LITTER SIZE ON SOME PRODUCTIVE PARAMETERS AND MILK COMPONENTS OF SHEEP UNDER SEMI - INTENSIVE BREEDING
}

\author{
Khalid. H. Sultan \\ Animal Production Dept. College of Agriculture and Forestry/ College of Agriculture \\ and Forestry/ Mosul University/ Iraq \\ Email: khalidhassani_1961@yahoo.com
}

\begin{abstract}
In the current study, 28 Awassi ewes (2-3 aged) years aged were used with their lambs for 12 weeks, to evaluate the effect of ewes body weight (BW) and Litter size (LS) on Body weight BW, Body gain (BG) of lambs, Milk yield (MY) and milk components of ewes. After lambing, ewes were weighted directly. Ewes distributed to 4 groups depending on it's body weight, the groups were: $1^{\text {st }}$ and $2^{\text {nd }}$ groups: ewes with high body weight and single lambing (HS), and twin lambing (HT) respectively, $3^{\text {rd }}$ and $4^{\text {th }}$ groups: ewes with low body weight and single lambing LS, and twin lambing LT respectively. The results showed a significant increase $(\mathrm{p} \leq 0.05)$ in BW and BG in HS lambs groups compared with LT lambs most weeks of study. MY was higher significantly $(p \leq 0.05)$ in Heavy ewes group that have twin lambs at most weeks of study, milk fat $\%$ recorded a significant increase in milk fat $\%$ in Heavy ewes group with single lamb. In conclusion, single lambs born and reared by heavy ewes were became heavier at the end of lactation period (at weaning), also ewes with twin lambs regardless if it was heavy or light will have more milk yield.
\end{abstract}

Key words: production, twin, milk traits, Awassi.

Received:18/9/2019, Accepted: 20/11/2019

\section{INTRODUCTION}

High lambing rate is the biggest contribute to get more profits from sheep farms. However, lamb's survivability is an important issue in highly fecund sheep flocks (8). In lambs meat production systems, ewes play a double role, they contribute directly to the number of lambs sold through their litter size, and indirectly, through the so - called maternal components, to the survival and growth of the lambs (Bardford,1972).Sheep farming has been developing, mainly due to the growth of production and consumption of high quality sheep meat (Sosa,2008), which is considered an attractive source of income to farmers. Many factors may influence the lambs development, which included year and type of birth, weight of dam and milk yield, in addition to feeding conditions (Acta and Dogan,2014; Abdul- Noor, 2002,; Gaskins, et al, 2005; Notter, et al, 2005 and Rashidi, et al, 2008). (Merkhan, (20019), reported a relation between body and udder measurements to be moderately and strongly associated with milk productions. This environmental factors had a significant effect on body weight of lambs at various ages through the available of space in uterine during pregnancy, nutrient supplies and competition for milk after birth especially with twins (Hasan and Seyed, 2009). Also birth and weaning weights of lambs were usually influenced by physiological, environmental and genetic factors, environmental factors including ewes age, litter size and growth type (Notter, et al, 2005). Milk is the sole source of nutrients for the 
newborn mammals, thus, its survival and potential to reach reproductive maturity are directly depend upon the lactational success of it's dam. Many environmental factors applied postnatally are known to affect milk production of the dam (Walker, et al, 2004 and Pulina, et al 2006).

The present study was undertaken to assess effect of body weight and litter size on some productive parameters and milk components of Awassi ewes.

\section{MATERIALS AND METHODS}

The study was carried out at Bibokht village which located northeast Mousl city, during the period $1 / 10 / 2017$ to $1 / 1 / 2018$, to evaluate the effect of body weight and litter size on some productive parameters of lambs, milk yield and components of ewes. The study include 28 pregnant Awassi ewes (2-3 years aged). The animals were housed in semi-open pens ( $24 \mathrm{~m}^{2}$ for each group), the animals were examined by the veterinarian and were healthy, disease-free and were supervised by veterinarians throughout the study period with all the required vaccines. Ewes divided randomly to 4 groups depending on it's body weight heavy $(44.73-46.17 \mathrm{~kg})$ or light $(36.22-37.15 \mathrm{~kg})$ and litter size single or twin lambing, the groups were: 1st group: ewes with high body weight and single lambing HS, 2nd group: ewes with high body weight and twin lambing HT,3rd group: ewes with low body weight and single lambing LS, $4^{\text {th }}$ group: ewes with low body weight and twin lambing LT. The concentrated ration was mentioned in (Table 4). Ration admitted to the animals at a rate of $1500 \mathrm{~g} / \mathrm{animal} / \mathrm{day}$, and the animals of each group were fed collectively, drinking water was freely available during the study period.

The amount of milk measured every 15 days twice daily at two consequentive days using manual milking method.

Table (1): Components and chemical analysis of the rations.

\begin{tabular}{|c|c|c|c|}
\hline \multicolumn{2}{|l|}{ Rations } & \multicolumn{2}{|c|}{ Chemical analysis } \\
\hline \multicolumn{2}{|l|}{ Ingredients $\%$} & \multicolumn{2}{|c|}{$* *$ Calculated chemical analysis\% } \\
\hline Barley & 50 & Dry matter & 90.22 \\
\hline Wheat bran & 21 & Organic matter & 93.10 \\
\hline Soybean meal * & 8 & Crud Fiber & 4.95 \\
\hline Yellow corn & 15 & Ether extract & 2.60 \\
\hline Urea & 0.5 & Crud protein & 14.13 \\
\hline Wheat straw & 4.5 & Dissolved carbohydrates & 70.10 \\
\hline Food salt & 0.5 & Ash & 6.90 \\
\hline Calcium Carbonate & 0.5 & $\mathrm{ME}(\mathrm{Kcal} / \mathrm{Kg} / \mathrm{DM})$ & 2538.00 \\
\hline
\end{tabular}

The milk was analyzed using an Eko-milk analyzer, the analysis includes fat, protein, lactose and non- solid fat\%. Data were analyzed using the General Liner Model (GLM) procedure (Anonymous, 2002) assuming the following model:

Yijk $=\mu+B i+L j+B L i j+e i j k$ 
Differences among means were tested using Duncan multiple rang test (Steel and Torrie, 1984).

\section{RESULTS AND DISCUSSION}

The results of the effect of body weight heavy $(\mathrm{H})$ or light $(\mathrm{L})$ and litter size single $(\mathrm{S})$ or $(\mathrm{T})$ on body weight $(\mathrm{BW})$ and body gain $(\mathrm{BG})$ of Awassi lambs are presented in (Table 2), whose showed a significant increase in BW of $\mathrm{H}$ and $\mathrm{S}$ lambs at most weeks of treatment, as compared with $\mathrm{L}$ and $\mathrm{T}$ lambs. Table 2 also revealed a significant increase $(\mathrm{P} \leq 0.05)$ in $\mathrm{BG}$ of $\mathrm{H}$ and $\mathrm{S}$ lambs at most weeks of treatment. Values of milk yield (gm), milk fat\% and milk protein\% of Awassi ewes are presented in (Table 3), and showed a significant increase in milk yield of $\mathrm{H}$ ewes as compared with $\mathrm{L}$ ewes, in most weeks of study, also there were a significant $(\mathrm{P} \leq 0.05)$ increase in milk yield at 4 th, 6th, 8th, and 12th weeks in $\mathrm{T}$ ewes as compared with $\mathrm{S}$ ewes. Milk fat\% increased significantly $(\mathrm{P} \leq 0.05)$ in $\mathrm{S}$ ewes as compared with $\mathrm{T}$ ewes, while

reported a significant $(\mathrm{P} \leq 0.05)$ in ewes milk as compared with $\mathrm{L}$ ewes at most weeks of study.

Table (2): Effect of ewes body weight and litter size on lambing and weaning Body weight $(\mathrm{kg})$ in Awassi sheep.

\begin{tabular}{|c|c|c|c|c|c|c|}
\hline \multirow[b]{3}{*}{$\begin{array}{c}\text { Body } \\
\text { weight }\end{array}$} & \multicolumn{6}{|c|}{ Weeks after birth/Litter size } \\
\hline & \multicolumn{2}{|c|}{2} & \multicolumn{2}{|c|}{4} & \multicolumn{2}{|c|}{6} \\
\hline & S & $\mathrm{T}$ & S & $\mathrm{T}$ & S & $\mathrm{T}$ \\
\hline $\mathrm{H}$ & $\begin{array}{r}4.26 \mathrm{a} \\
\pm 0.13\end{array}$ & $\begin{array}{l}3.57 \mathrm{~b} \\
\pm 0.16\end{array}$ & $\begin{array}{r}6.85 \mathrm{a} \\
\pm 0.14\end{array}$ & $\begin{array}{r}6.00 \mathrm{~b} \\
\pm 0.13\end{array}$ & $\begin{array}{l}8.61 \mathrm{a} \\
\pm 0.25\end{array}$ & $\begin{array}{l}8.52 \mathrm{a} \\
\pm 0.23\end{array}$ \\
\hline \multirow[t]{2}{*}{$\mathrm{L}$} & $\begin{array}{l}3.57 \mathrm{~b} \\
\pm 0.16\end{array}$ & $\begin{array}{l}3.13 \mathrm{~b} \\
\pm 0.14\end{array}$ & $\begin{array}{r}5.80 \mathrm{~b} \\
\pm 0.14 \\
\end{array}$ & $\begin{array}{r}5.72 \mathrm{~b} \\
\pm 0.14 \\
\end{array}$ & $\begin{array}{r}8.18 \mathrm{a} \\
\pm 0.22 \\
\end{array}$ & $\begin{array}{r}7.13 \mathrm{~b} \\
\pm 0.22 \\
\end{array}$ \\
\hline & \multicolumn{6}{|c|}{ Weeks/Litter size } \\
\hline \multirow[b]{2}{*}{$\begin{array}{c}\text { Body } \\
\text { weight }\end{array}$} & \multicolumn{2}{|c|}{8} & \multicolumn{2}{|c|}{10} & \multicolumn{2}{|c|}{12} \\
\hline & $\mathrm{S}$ & $\mathrm{T}$ & $\mathrm{S}$ & $\mathrm{T}$ & $\mathrm{S}$ & $\mathrm{T}$ \\
\hline $\mathrm{H}$ & $\begin{array}{c}15.98 \mathrm{a} \\
\pm 0.19 \\
\end{array}$ & $\begin{array}{c}13.77 \mathrm{~b} \\
\pm 0.45\end{array}$ & $\begin{array}{c}19.50 \mathrm{a} \\
\pm 0.23 \\
\end{array}$ & $\begin{array}{r}18.85 b \\
\pm 0.26 \\
\end{array}$ & $\begin{array}{c}23.57 \mathrm{a} \\
\pm 0.19 \\
\end{array}$ & $\begin{array}{c}21.64 \mathrm{~b} \\
\pm 0.29 \\
\end{array}$ \\
\hline $\mathrm{L}$ & $\begin{array}{r}12.75 b \\
\pm 0.37\end{array}$ & $\begin{array}{c}13.69 \mathrm{~b} \\
\pm 0.53\end{array}$ & $\begin{array}{r}17.87 \mathrm{~b} \\
\pm 0.43\end{array}$ & $\begin{array}{r}17.88 b \\
\pm 0.35\end{array}$ & $\begin{array}{c}20.55 c \\
\pm 0.39\end{array}$ & $\begin{array}{r}20.40 \mathrm{c} \\
\pm 0.38\end{array}$ \\
\hline
\end{tabular}

Different litters vertically and horizontally in the same week marks significant differs at $\quad(\mathrm{P} \leq 0.05)$.

S: single lambing, T: twin lambing, H: heavy, L: light.

Values represent: mean $\pm \mathrm{SE}$.

No significant effects were showed of body weight and litter size on milk protein\% in all weeks of study except in 8 th and $10^{\text {th }}$ weeks, there were a significant increase in $\mathrm{H}$ ewes as compared with $\mathrm{L}$ ewes and in $\mathrm{T}$ ewes as compared with $\mathrm{S}$ ewes. And in regard to milk lactose \%, ewes with LS had significantly higher lactose\% than ewes with LT at the 4th and 8th weeks of lactation, and higher milk lactose\% was recorded in ewes with LT than in LS ewes at the 12th week of lactation, on the other hand, LS ewes had significantly higher milk lactose $\%$ as compared with HS ewes at $(\mathrm{P} \leq 0.05)$. For non- fat 
solid\% a significant increase in NFS in LS as compared with HS ewes and in LS ewes as compared with LT ewes at the 2nd week of lactation, and in HT ewes as compared with LT ewes at 6 th week of lactation.

Single lambs heavier at lambing than twins, because of the reduced uterine space limits variance in birth weight (Gluckman and Hanson, 2004). Birth type (single or twin) affect the weights of lambs at lambing to weaning due to the single born lambs had better opportunities in the mother's wombs than the twins or triplets and were hence heavy at birth. (Babar, et al, 2003).

Live weight of lambs at birth increased in proportion to the increase of Live weight of ewes (Akta and Dogan, 2014). Results were in agreement with the results of (Aliyari, et al, 2012) who reported that ewes with the highest body weights produced lambs with highest body weights, this may be attributed to the better development of mammary (Santello, 2008), whose reported a significant increase in body weight and body gain at birth till weaning in Hampshire Down lambs. Twins present lighter weights at birth because of gland in heavy ewes than light ewes. The impact of litter size of current study are in agreement with the results of Barrose, et al (2005) and an intra-uterine competition. Single lamb's BW and BG were more than twins, because twins receive less milk than singles, Kalantar, (2003) and Matika et al, (2003), reported that type of birth have no significant effect on BW and BG. Also results of BW and BG were in agreement with the results of Saghi, et al, (2007), who reported that single borne lambs have higher birth weight and daily growth in comparison with multiple born lambs. Heavy ewes born lambs heavier than lambs born from light ewes, the reason of that may be in the body fat metabolism serving as a source for more milk yield in the heavy ewes (Corner et al, 2013). Heavy and twin lambing ewes produced more milk than light and single lambing ewes, this results was in agreement with Abdul- Noor, et al,2002; Al-Azzawi and Al-Rawi, 1997 and Kassem et al, 2010, whom reported a significant increase in milk yield with high twinning rates, this increase attributed to the higher stimulation of twins rather than single lambing. Orhan et al, (2011), report no effects of birth type on milk fat, protein, lactose and total solids\%.

In conclusion, body weights and body gain lambs, milk yield, milk fat $\%$ and milk protein $\%$, increased significantly by the effect of the body weight and litter size. Heavy and twins lambing results more milk yield, while single lambs have a heavier weaning weights. 
Table (3): Effect of ewes body weight and litter size in lambing and weaning Body gain $(\mathrm{kg})$ of Awassi lambs.

\begin{tabular}{|c|c|c|c|c|c|c|}
\hline \multirow[b]{3}{*}{$\begin{array}{c}\text { Body } \\
\text { weight }\end{array}$} & \multicolumn{6}{|c|}{ Weeks after birth/Litter size } \\
\hline & \multicolumn{2}{|c|}{2} & \multicolumn{2}{|c|}{4} & \multicolumn{2}{|c|}{6} \\
\hline & $\mathrm{S}$ & $\mathrm{T}$ & $\mathrm{S}$ & $\mathrm{T}$ & $\mathrm{S}$ & $\mathrm{T}$ \\
\hline $\mathrm{H}$ & $\begin{array}{l}2.59 \mathrm{a} \\
\pm 0.25\end{array}$ & $\begin{array}{l}2.86 \mathrm{a} \\
\pm 0.14\end{array}$ & $\begin{array}{l}1.75 \mathrm{~b} \\
\pm 0.14\end{array}$ & $\begin{array}{l}2.52 \mathrm{a} \\
\pm 0.17\end{array}$ & $\begin{array}{l}3.44 \mathrm{a} \\
\pm 0.16\end{array}$ & $\begin{array}{l}2.48 \mathrm{~b} \\
\pm 0.21\end{array}$ \\
\hline \multirow[t]{2}{*}{ L } & $\begin{array}{c}2.22 \mathrm{~b} \pm \\
0.12\end{array}$ & $\begin{array}{c}2.59 \mathrm{~b} \pm \\
0.14\end{array}$ & $\begin{array}{l}3.38 \mathrm{a} \\
\pm 0.17\end{array}$ & $\begin{array}{l}1.40 \mathrm{~b} \\
\pm 0.15\end{array}$ & $\begin{array}{l}2.78 \mathrm{~b} \\
\pm 0.15\end{array}$ & $\begin{array}{l}2.75 \mathrm{~b} \\
\pm 0.18\end{array}$ \\
\hline & \multicolumn{6}{|c|}{ Weeks/Litter size } \\
\hline \multirow[b]{2}{*}{$\begin{array}{c}\text { Body } \\
\text { weight }\end{array}$} & \multicolumn{2}{|c|}{8} & \multicolumn{2}{|c|}{10} & \multicolumn{2}{|c|}{12} \\
\hline & $\mathrm{S}$ & $\mathrm{T}$ & $\mathrm{S}$ & $\mathrm{T}$ & $\mathrm{S}$ & $\mathrm{T}$ \\
\hline $\mathrm{H}$ & $\begin{array}{l}3.92 \mathrm{a} \\
\pm 0.14\end{array}$ & $\begin{array}{c}2.75 \mathrm{~b} \pm \\
0.30\end{array}$ & $\begin{array}{l}3.51 \mathrm{~b} \\
\pm 0.21\end{array}$ & $\begin{array}{l}5.07 \mathrm{a} \\
\pm 0.39\end{array}$ & $\begin{array}{l}4.07 \mathrm{a} \\
\pm 0.30\end{array}$ & $\begin{array}{l}2.79 \mathrm{~b} \\
\pm 0.24\end{array}$ \\
\hline $\mathrm{L}$ & $\begin{array}{l}1.78 \mathrm{~b} \\
\pm 0.20\end{array}$ & $\begin{array}{l}3.80 \mathrm{a} \\
\pm 0.38\end{array}$ & $\begin{array}{r}5.11 \mathrm{a} \\
\pm .28\end{array}$ & $\begin{array}{l}4.19 \mathrm{~b} \\
\pm 0.42\end{array}$ & $\begin{array}{l}2.68 \mathrm{~b} \\
\pm 0.14\end{array}$ & $\begin{array}{l}2.52 \mathrm{~b} \\
\pm 0.14\end{array}$ \\
\hline
\end{tabular}

Different litters vertically and horizontally in the same week marks significant differs at $\quad(\mathrm{P} \leq 0.05)$. S: single lambing, T: twin lambing, H: heavy, L: light.

Values represent: mean $\pm \mathrm{SE}$.

Table (4): Effect of ewes body weight and litter size on Milk yield (gm) of Awassi sheep.

\begin{tabular}{|c|c|c|c|c|c|c|}
\hline \multirow[b]{3}{*}{$\begin{array}{c}\text { Body } \\
\text { weight }\end{array}$} & \multicolumn{6}{|c|}{ Weeks after birth/Litter size } \\
\hline & \multicolumn{2}{|c|}{2} & \multicolumn{2}{|c|}{4} & \multicolumn{2}{|c|}{6} \\
\hline & S & $\mathrm{T}$ & S & $\mathrm{T}$ & S & $\mathrm{T}$ \\
\hline $\mathrm{H}$ & $\begin{array}{l}791.43 \mathrm{a} \\
\pm 14.82\end{array}$ & $\begin{array}{c}802.86 \mathrm{a} \\
\pm 22.64\end{array}$ & $\begin{array}{c}797.14 \mathrm{~b} \\
\pm 17.95\end{array}$ & $\begin{array}{c}902.86 \mathrm{a} \\
\pm 21.57\end{array}$ & $\begin{array}{c}867.86 \mathrm{~b} \\
\pm 12.62\end{array}$ & $\begin{array}{l}970.00 \mathrm{a} \\
\pm 12.14\end{array}$ \\
\hline \multirow[t]{2}{*}{$\mathrm{L}$} & $\begin{array}{l}501.43 \mathrm{~b} \\
\pm 34.32 \\
\end{array}$ & $\begin{array}{c}460.00 \mathrm{~b} \\
\pm 21.60 \\
\end{array}$ & $\begin{array}{c}547.86 \mathrm{c} \\
\pm 11.84 \\
\end{array}$ & $\begin{array}{c}550.71 \mathrm{c} \\
\pm 31.63 \\
\end{array}$ & $\begin{array}{l}532.86 \mathrm{c} \\
\pm 14.75 \\
\end{array}$ & $\begin{array}{l}541.43 \mathrm{c} \\
\pm 10.10 \\
\end{array}$ \\
\hline & \multicolumn{6}{|c|}{ Weeks/Litter size } \\
\hline \multirow[b]{2}{*}{$\begin{array}{c}\text { Body } \\
\text { weight }\end{array}$} & \multicolumn{2}{|c|}{8} & \multicolumn{2}{|c|}{10} & \multicolumn{2}{|c|}{12} \\
\hline & S & $\mathrm{T}$ & S & $\mathrm{T}$ & S & $\mathrm{T}$ \\
\hline $\mathrm{H}$ & $\begin{array}{c}752.86 \mathrm{~b} \\
\pm 12.67 \\
\end{array}$ & $\begin{array}{c}920 . \mathrm{a} a \\
\pm 6.17 \\
\end{array}$ & $\begin{array}{c}822.14 \mathrm{a} \\
\pm 14.87 \\
\end{array}$ & $\begin{array}{c}850.71 \mathrm{a} \\
\pm 22.74 \\
\end{array}$ & $\begin{array}{c}735.00 \mathrm{~b} \\
\pm 19.66 \\
\end{array}$ & $\begin{array}{l}855.00 \mathrm{a} \\
\pm 13.75 \\
\end{array}$ \\
\hline $\mathrm{L}$ & $\begin{array}{c}508.57 \mathrm{c} \\
\pm 16.24\end{array}$ & $\begin{array}{c}490.00 \mathrm{c} \\
\pm 22.88\end{array}$ & $\begin{array}{c}450.00 \mathrm{c} \\
\pm 26.72\end{array}$ & $\begin{array}{c}532.00 \mathrm{~b} \\
\pm 20.29\end{array}$ & $\begin{array}{c}400.71 \mathrm{a} \\
\pm 22.50\end{array}$ & $\begin{array}{l}520.00 \mathrm{c} \\
\pm 20.90\end{array}$ \\
\hline
\end{tabular}

Different litters vertically and horizontally in the same week marks significant differs at $(\mathrm{P} \leq 0.05) . \mathrm{S}$ : single lambing, T: twin lambing, $\mathrm{H}$ : heavy, L: light. 
Table (5): Effect of ewes body weight and litter size on Milk fat percentage .

\begin{tabular}{|c|c|c|c|c|c|c|}
\hline \multirow[b]{3}{*}{$\begin{array}{c}\text { Body } \\
\text { weight }\end{array}$} & \multicolumn{6}{|c|}{ Weeks after birth/Litter size } \\
\hline & \multicolumn{2}{|c|}{2} & \multicolumn{2}{|c|}{4} & \multicolumn{2}{|c|}{6} \\
\hline & $\mathrm{S}$ & $\mathrm{T}$ & $\mathrm{S}$ & $\mathrm{T}$ & $\mathrm{S}$ & $\mathrm{T}$ \\
\hline $\mathrm{H}$ & $\begin{array}{l}4.58 \mathrm{a} \\
\pm 0.11\end{array}$ & $\begin{array}{l}3.68 \mathrm{~b} \\
\pm 0.13\end{array}$ & $\begin{array}{l}4.49 \mathrm{a} \\
\pm 0.13\end{array}$ & $\begin{array}{l}4.45 \mathrm{a} \\
\pm 0.12\end{array}$ & $\begin{array}{r}4.62 \mathrm{ab} \\
\pm 0.22\end{array}$ & $\begin{array}{l}4.00 \mathrm{bc} \\
\pm 0.19\end{array}$ \\
\hline \multirow[t]{2}{*}{ L } & $\begin{array}{l}3.68 \mathrm{~b} \\
\pm 0.13\end{array}$ & $\begin{array}{l}3.34 \mathrm{~b} \\
\pm 0.14\end{array}$ & $\begin{array}{l}3.73 \mathrm{~b} \\
\pm 0.18\end{array}$ & $\begin{array}{l}4.05 \mathrm{~b} \\
\pm 0.20\end{array}$ & $\begin{array}{l}4.73 \mathrm{a} \\
\pm 0.15\end{array}$ & $\begin{array}{l}3.86 \mathrm{c} \\
\pm 0.21\end{array}$ \\
\hline & \multicolumn{6}{|c|}{ Weeks/Litter size } \\
\hline \multirow[b]{2}{*}{$\begin{array}{c}\text { Body } \\
\text { weight }\end{array}$} & \multicolumn{2}{|c|}{8} & \multicolumn{2}{|c|}{10} & \multicolumn{2}{|c|}{12} \\
\hline & $\mathrm{S}$ & $\mathrm{T}$ & $\mathrm{S}$ & $\mathrm{T}$ & $\mathrm{S}$ & $\mathrm{T}$ \\
\hline $\mathrm{H}$ & $\begin{array}{l}4.54 \mathrm{a} \\
\pm 0.10\end{array}$ & $\begin{array}{l}4.00 \mathrm{~b} \\
\pm 0.03\end{array}$ & $\begin{array}{l}4.73 \mathrm{a} \\
\pm 0.09\end{array}$ & $\begin{array}{l}3.89 \mathrm{~b} \\
\pm 0.11\end{array}$ & $\begin{array}{l}4.58 \mathrm{a} \\
\pm 0.16\end{array}$ & $\begin{array}{l}3.97 \mathrm{~b} \\
\pm 0.06\end{array}$ \\
\hline $\mathrm{L}$ & $\begin{array}{l}4.44 \mathrm{a} \\
\pm 0.12\end{array}$ & $\begin{array}{l}3.94 \mathrm{~b} \\
\pm 0.08\end{array}$ & $\begin{array}{l}4.91 \mathrm{a} \\
\pm 0.10\end{array}$ & $\begin{array}{l}4.09 \mathrm{~b} \\
\pm 0.03\end{array}$ & $\begin{array}{l}4.11 b \\
\pm 0.06\end{array}$ & $\begin{array}{l}4.04 \mathrm{~b} \\
\pm 0.05\end{array}$ \\
\hline
\end{tabular}

Different litters vertically and horizontally in the same week marks significant differs at $\quad(\mathrm{P} \leq 0.05)$.

S: single lambing, T: twin lambing, H: heavy, L: light.

Table (6): Effect of ewes body weight and litter size on Milk protein percentage .

\begin{tabular}{|c|c|c|c|c|c|c|}
\hline \multirow{3}{*}{$\begin{array}{c}\text { Body } \\
\text { weight }\end{array}$} & \multicolumn{6}{|c|}{ Weeks after birth/Litter size } \\
\hline & \multicolumn{2}{|c|}{2} & \multicolumn{2}{|c|}{4} & \multicolumn{2}{|c|}{6} \\
\hline & S & $\mathrm{T}$ & S & $\mathrm{T}$ & S & $\mathrm{T}$ \\
\hline $\mathrm{H}$ & $\begin{array}{l}4.46 \mathrm{a} \\
\pm 0.12\end{array}$ & $\begin{array}{l}4.64 \mathrm{a} \\
\pm 0.13\end{array}$ & $\begin{array}{l}4.62 \mathrm{a} \\
\pm 0.10\end{array}$ & $\begin{array}{l}4.76 \mathrm{a} \\
\pm 0.08\end{array}$ & $\begin{array}{l}4.41 \mathrm{a} \\
\pm 0.13\end{array}$ & $\begin{array}{l}4.47 \mathrm{a} \\
\pm 0.09\end{array}$ \\
\hline \multirow[t]{2}{*}{$\mathrm{L}$} & $\begin{array}{l}4.52 \mathrm{a} \\
\pm 0.08\end{array}$ & $\begin{array}{l}4.31 \mathrm{a} \\
\pm 0.13\end{array}$ & $\begin{array}{l}4.68 \mathrm{a} \\
\pm 0.21\end{array}$ & $\begin{array}{l}4.61 \mathrm{a} \\
\pm 0.09\end{array}$ & $\begin{array}{l}4.41 \mathrm{a} \\
\pm 0.09\end{array}$ & $\begin{array}{l}4.42 \mathrm{a} \\
\pm 0.07\end{array}$ \\
\hline & \multicolumn{6}{|c|}{ Weeks/Litter size } \\
\hline \multirow[b]{2}{*}{$\begin{array}{c}\text { Body } \\
\text { weight }\end{array}$} & \multicolumn{2}{|c|}{8} & \multicolumn{2}{|c|}{10} & \multicolumn{2}{|c|}{12} \\
\hline & $\mathrm{S}$ & $\mathrm{T}$ & $\mathrm{S}$ & $\mathrm{T}$ & $\mathrm{S}$ & $\mathrm{T}$ \\
\hline $\mathrm{H}$ & $\begin{array}{c}4.45 \mathrm{ab} \\
\pm 0.11\end{array}$ & $\begin{array}{l}4.76 \mathrm{a} \\
\pm 0.16\end{array}$ & $\begin{array}{l}4.35 \mathrm{~b} \\
\pm 0.06\end{array}$ & $\begin{array}{l}4.66 \mathrm{a} \\
\pm 0.07\end{array}$ & $\begin{array}{l}4.58 \mathrm{a} \\
\pm 0.08\end{array}$ & $\begin{array}{l}4.46 \mathrm{a} \\
\pm 0.08\end{array}$ \\
\hline $\mathrm{L}$ & $\begin{array}{l}4.55 \mathrm{ab} \\
\pm 0.15\end{array}$ & $\begin{array}{l}4.30 \mathrm{~b} \\
\pm 0.11\end{array}$ & $\begin{array}{r}4.48 \mathrm{ab} \\
\pm 0.09\end{array}$ & $\begin{array}{l}4.49 \mathrm{ab} \\
\pm 0.09\end{array}$ & $\begin{array}{l}4.50 \mathrm{a} \\
\pm 0.10\end{array}$ & $\begin{array}{l}4.50 \mathrm{a} \\
\pm 0.10\end{array}$ \\
\hline
\end{tabular}

Different litters vertically and horizontally in the same week marks significant differs at $(\mathrm{P} \leq 0.05)$.

S: single lambing, T: twin lambing, H: heavy, L: light. 
Table (7): Effect of ewes body weight and litter size Milk lactose percentage .

\begin{tabular}{|c|c|c|c|c|c|c|}
\hline \multirow[b]{3}{*}{$\begin{array}{c}\text { Body } \\
\text { weight }\end{array}$} & \multicolumn{6}{|c|}{ Weeks after birth/Litter size } \\
\hline & \multicolumn{2}{|c|}{2} & \multicolumn{2}{|c|}{4} & \multicolumn{2}{|c|}{6} \\
\hline & $\mathrm{S}$ & $\mathrm{T}$ & $\mathrm{S}$ & $\mathrm{T}$ & $\mathrm{S}$ & $\mathrm{T}$ \\
\hline $\mathrm{H}$ & $\begin{array}{l}4.46 \mathrm{a} \\
\pm 0.04\end{array}$ & $\begin{array}{l}4.44 \mathrm{a} \\
\pm 0.08\end{array}$ & $\begin{array}{c}4.45 \mathrm{ab} \\
\pm 0.06\end{array}$ & $\begin{array}{c}4.33 \mathrm{ab} \\
\pm 0.07\end{array}$ & $\begin{array}{l}4.45 \mathrm{a} \\
\pm 0.10\end{array}$ & $\begin{array}{l}4.56 \mathrm{a} \\
\pm 0.09\end{array}$ \\
\hline \multirow[t]{2}{*}{ L } & $\begin{array}{l}4.59 \mathrm{a} \\
\pm 0.10\end{array}$ & $\begin{array}{l}4.41 \mathrm{a} \\
\pm 0.09\end{array}$ & $\begin{array}{l}4.41 \mathrm{a} \\
\pm 0.14\end{array}$ & $\begin{array}{l}4.22 \mathrm{~b} \\
\pm 0.03\end{array}$ & $\begin{array}{l}4.39 \mathrm{a} \\
\pm 0.09\end{array}$ & $\begin{array}{c}3.79 \mathrm{a} \\
\pm .55\end{array}$ \\
\hline & \multicolumn{6}{|c|}{ Weeks/Litter size } \\
\hline \multirow[b]{2}{*}{$\begin{array}{c}\text { Body } \\
\text { weight }\end{array}$} & \multicolumn{2}{|c|}{8} & \multicolumn{2}{|c|}{10} & \multicolumn{2}{|c|}{12} \\
\hline & $\mathrm{S}$ & $\mathrm{T}$ & $\mathrm{S}$ & $\mathrm{T}$ & $\mathrm{S}$ & $\mathrm{T}$ \\
\hline $\mathrm{H}$ & $\begin{array}{l}4.33 \mathrm{~b} \\
\pm 0.09\end{array}$ & $\begin{array}{l}4.42 \mathrm{~b} \\
\pm 0.09\end{array}$ & $\begin{array}{l}4.36 \mathrm{a} \\
\pm 0.10\end{array}$ & $\begin{array}{l}4.49 \mathrm{a} \\
\pm 1.10\end{array}$ & $\begin{array}{l}4.50 \mathrm{a} \\
\pm 0.09\end{array}$ & $\begin{array}{c}4.37 \mathrm{ab} \\
\pm 0.11\end{array}$ \\
\hline $\mathrm{L}$ & $\begin{array}{l}4.72 \mathrm{a} \\
\pm 0.09\end{array}$ & $\begin{array}{l}4.37 \mathrm{~b} \\
\pm 0.08\end{array}$ & $\begin{array}{l}4.46 \mathrm{a} \\
\pm 0.11\end{array}$ & $\begin{array}{l}4.45 \mathrm{a} \\
\pm 0.09\end{array}$ & $\begin{array}{l}4.21 \mathrm{~b} \\
\pm 0.05\end{array}$ & $\begin{array}{l}4.55 \mathrm{a} \\
\pm 0.09\end{array}$ \\
\hline
\end{tabular}

Different litters vertically and horizontally in the same week marks significant differs at $\quad(\mathrm{P} \leq 0.05)$.

S: single lambing, T: twin lambing, H: heavy, L: light.

Table (8): Effect of ewes body weight and litter size in Solid non fat percentage.

\begin{tabular}{|c|c|c|c|c|c|c|}
\hline \multirow[b]{3}{*}{$\begin{array}{l}\text { Body } \\
\text { weight }\end{array}$} & \multicolumn{6}{|c|}{ Weeks after birth/Litter size } \\
\hline & \multicolumn{2}{|c|}{2} & \multicolumn{2}{|c|}{4} & \multicolumn{2}{|c|}{6} \\
\hline & S & $\mathrm{T}$ & $\mathrm{S}$ & $\mathrm{T}$ & S & $\mathrm{T}$ \\
\hline $\mathrm{H}$ & $\begin{array}{c}10.37 \mathrm{~b} \\
\pm 0.08 \\
\end{array}$ & $\begin{array}{c}10.56 \mathrm{ab} \\
\pm 0.10\end{array}$ & $\begin{array}{l}10.80 \mathrm{a} \\
\pm 0.18\end{array}$ & $\begin{array}{c}10.74 \mathrm{a} \\
\pm 0.19\end{array}$ & $\begin{array}{c}10.77 \mathrm{ab} \\
\pm 0.07\end{array}$ & $\begin{array}{l}11.03 \mathrm{a} \\
\pm 0.22\end{array}$ \\
\hline \multirow[t]{2}{*}{$\mathrm{L}$} & $\begin{array}{c}10.80 \mathrm{a} \\
\pm 0.08\end{array}$ & $\begin{array}{c}10.43 \mathrm{~b} \\
\pm 0.10\end{array}$ & $\begin{array}{c}10.46 \mathrm{a} \\
\pm 0.13\end{array}$ & $\begin{array}{r}10.51 \mathrm{a} \\
\pm 0.11\end{array}$ & $\begin{array}{c}10.44 \mathrm{~b} \\
\pm 0.13\end{array}$ & $\begin{array}{c}10.47 \mathrm{~b} \\
\pm 0.13\end{array}$ \\
\hline & \multicolumn{6}{|c|}{ Weeks after /Litter size } \\
\hline \multirow[b]{2}{*}{$\begin{array}{c}\text { Body } \\
\text { weight }\end{array}$} & \multicolumn{2}{|c|}{8} & \multicolumn{2}{|c|}{10} & \multicolumn{2}{|c|}{12} \\
\hline & $\mathrm{S}$ & $\mathrm{T}$ & S & $\mathrm{T}$ & $\mathrm{S}$ & $\mathrm{T}$ \\
\hline $\mathrm{H}$ & $\begin{array}{c}10.76 \mathrm{a} \pm \\
0.13\end{array}$ & $\begin{array}{c}10.74 \mathrm{a} \pm \\
0.36\end{array}$ & $\begin{array}{c}10.46 \mathrm{a} \pm \\
0.13\end{array}$ & $\begin{array}{l}10.72 \mathrm{a} \\
\pm 0.12 \\
\end{array}$ & $\begin{array}{c}10.64 \mathrm{a} \pm \\
0.20\end{array}$ & $\begin{array}{c}10.92 \mathrm{a} \pm \\
0.16\end{array}$ \\
\hline $\mathrm{L}$ & $\begin{array}{c}10.54 \mathrm{a} \pm \\
0.13\end{array}$ & $\begin{array}{c}10.62 \mathrm{a} \pm \\
0.13\end{array}$ & $\begin{array}{c}10.74 \mathrm{a} \\
\pm 0.27\end{array}$ & $\begin{array}{r}10.68 \mathrm{a} \\
\pm 0.12 \\
\end{array}$ & $\begin{array}{c}10.59 \mathrm{a} \pm \\
0.12\end{array}$ & $\begin{array}{c}10.70 \mathrm{a} \pm \\
0.07\end{array}$ \\
\hline
\end{tabular}

Different litters vertically and horizontally in the same week marks significant differs at $(\mathrm{P} \leq 0.05)$.S: single lambing, $\mathrm{T}$ : twin lambing, $\mathrm{H}$ : heavy, L: light. 


\title{
تأثير وزن الجسم ونوع الولادة في بعض الصفات الانتاجية ومكونات الحليب في الأغنام تحت ظروف التربية شبه المكثقة \\ قسم الإنتاج الحيو اني_كلية الزر اعة والغاني سلطان_-جامعة الموصل/العراق \\ Email: khalidhassani_1961@yahoo.com
}

\begin{abstract}
الخلاصة

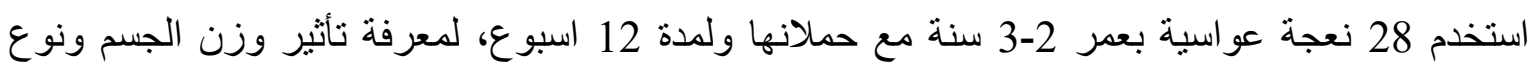

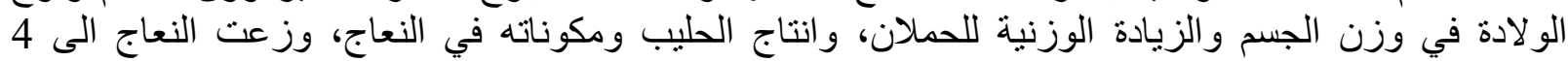

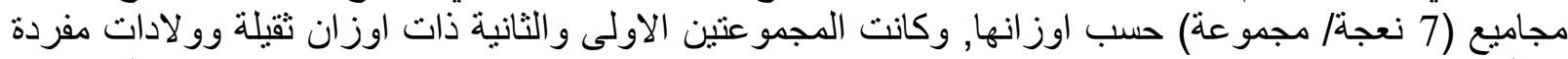

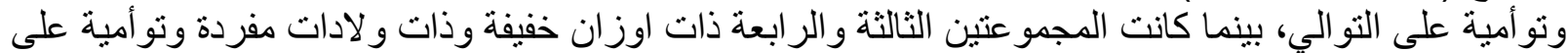

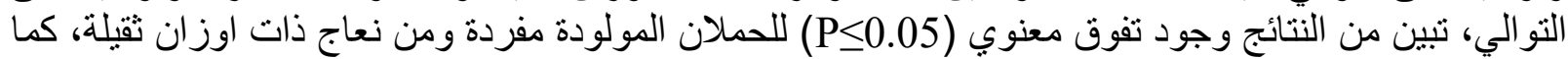

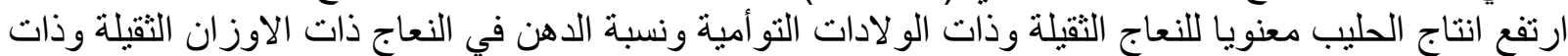

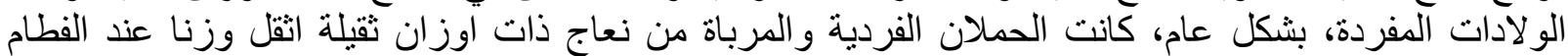

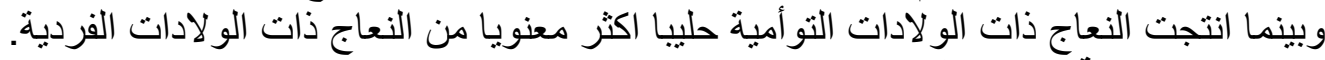

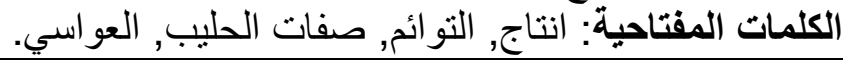

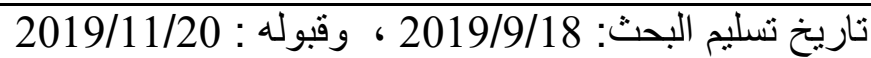

\section{REFERENCES}

Abdul-Noor,M. (2002). Study of some Factors in Milk yield and Lactation Period in Local and Turkish Awassi sheep. Diyala Journal of Animal Sciences. 3 (1) : 21 29.

Al-Azzawi, W.A and Al-Rawi,A.A. (1997). Repeteatability estimates of some economic productive traits in Awassi sheeo.IPA.J.Agri.Res.7(1):74-86.

Akta,s, A. H. and Do gan, , S. (2014). Effect of live weight and age of Akkaraman ewes at mating on multiple birth rate, growth traits, and survival rate of lambs, Turk. J. Vet. Anim. Sci., 38, 176-182.

Al-khawaja, A.K., Elham, A., and Abdul-Ahad,S. (1978).Chemical composition and nutritional value of Iraqi feed materials. $3^{\text {rd }}$ Edition. Ministry of Agriculture and Agrarian Reform, Directorate of Public Livestock, Iraq.

Aliyari, D., Moeini, M. M., Shahir, M. H., and Sirjani, M. A. (2012). Effect of BSC, live weight and age on reproductive performance of Afshari Ewes, Asian J. Anim. Vet. Adv., 7, 904-909.

Babar, M. E. Ahmad, Z. Nadeem, A. and Yaqoob, M. (2003). Enviromental Factors Affecting Weaning Weight in Lohi Sheep.Pakistan Vet. J., 23 (4): 177-180.

Barrose, N.N.; Vasconcelos, V.R. and Wander, A.E. • (2005) EficiênciabioeconômicadecordeirosF1DorperxSantaInêsparaprodução de carne. Pesquisa .Agropecuária Brasileira,40, (8). p.825-831.

Binns SH, Cox IJ, Rizvi S, Green LE.(2002) Risk factors of lamb mortality of UK sheep farms . Prev Vet Med; 52: 287-303.

Bardford G.E.,(1972) The role of maternal effects in animal breeding: VII - Maternal effects in sheep, J. Anim. Sci. 35 1324-1334.

Corner, R. A., Mulvaney, F. J., Morris, S. T., West, D. M., Morel, P. C. H., an Kenyon, P. R. (2013). A comparison of the reproductive performance of ewe lambs and mature ewes, Small Rumin. Res., 114, 126-133. 
Demirel, M., Kurbal, O. F., Aygun, T., Erdogan, S., Bakici, Y., Yılmaz, A., and Ulker, H. (2004). Effects of different feeding levels during mating period on the reproductive performance of Norduz ewes and growth and survival rate of their lambs, J. Biol. Sci., 4, 283- 287.

Gaskins, C. T., Snowder, G. D., Westman, M. K., and Evans, M.(2005).Influence of body weight, age and weight gain on fertility and prolificacy in four breeds of ewe lambs, J. Anim. Sci., 83, $1680-1689$.

Gluckman, P.D. and Hanson, M.A.(2004). Maternal constraint of fetal growth and its consequences. Seminars in Fetal \& Neonatal Medicine. 9, 419-425.

Hasan B., and Seyed H., H. (2009). Effects of environmental factors on growth traits in Ghezel sheep. African Journal of Biotechnology Vol. 8 (12), pp. 2903-2907, 17 June.

Kalantar M 2003. Evalution of some environmental effect on Growth traits in Zandi sheep. Agric. Res. 4: 49-58.

Kassem, R. Walid, A. Khalil,A. Yasin, M. Solieman,S Ziad, A. Ismail, E.Hussain, O and Mustafa, S. (2010). Factors Influencing the Milk Production of Awassi Sheep in A Flock With the Selected Lines at the Agricultural Scientific Research Centre in Salamieh/Syria. Kafkas Univ Vet Fak Derg. 16 (3): 425-430.

Kassem R. (1988). The Awassi sheep breeding project in Syria. Increasing small ruminant productivity in Semi-arid Areas. ICARDA, 155-163.

Matika, O, Van Wyk JB, Erasmus GJ, Baker RL (2003). Genetic parameter estimates in Sabi sheep. Livest. Prod. Sci. 79: 17-28.

Merkhan, K. Y. (2019). milk production and body weight at weaning and their relationships with body and udder measurements in meriz goats. Iraqi Journal of Agricultural Sciences. :50(1), 080-084.

Natália, A, Koritiaki1, E. L Azambuja, R, Ivone Y. M, Leandro, D. S, M A, Alves F B, Valter H, B. J, Filipe, A, B. C and Camila C. 2013.Influence of environmental factors onponderal performance and morphometric characteristics of lambs of different genetic groups from birth to weaning. R. Bras. Zootec., 42; (7), PP .463-470.

Notter, D. R., Borg, R. C., and Kuehn, L. A.: (2005) Adjustment of lamb birth and weaning weights for continuous effects of ewe age, Anim. Sci., 80, 241-248.

Orhan, Y. Bahattin, C. and Memis, B. (2011). Eff ects of Lactation Stage, Age, Birth Type and Body Weight on Chemical Composotion of Red Karaman Sheep Milk. Kafkas Univ Vet Fak Derg17 (3): 383-386.

Pulina, G., A. Nudda, G. Battacone, and A. Cannas. (2006). Effects of nutrition on the contents of fat, protein, somatic cells, aromatic compounds, and undesirable substances in sheep milk. Anim. Feed Sci. Technol. 131:255-291.

Saghi, D.A. Kh, H. Navidzadeh, M. and Nikbakhti, M. (2007). Study on Influence of Environmental Effect on Birth Weight, Weaning Weight and Daily Growth of Baluchi Sheep). Pakistan Journal of Nutrition 6 (5): 436-437.

Anonymous, SAS. (2002). Statistical Analysis systems. Software, v.9, SAS Institute, Cary, NC.

Shahroudi Eftekhar F, Shiri A, Twakolyan J, Danesh Mesgaran M. (2003). Estimation of maternal effects on growth traits of Kurdish lambs in north of khorasan. Pjoohesh Sazandegi. 50: 62-66. 
Steel, R. G. D. and J. H. Torrie. (1984). Principles and procedures of statistics. 2nd Ed., McGraw-Hill Co., New York, USA

Rashidi, A.; Mokhtari, M.S.; jahanshahi, A.S. .(2008). Genetic parameter estimates of pre-weaning growth traits in Kermani sheep. Small Ruminant Research, 74, pp.165-171.

Santello, G.A. Desempenho.(2008) características das fibrasmusculares e das carcaças de cordeiros nascidos de ovelhas suplementadas com diferentes níveis de proteína. 87f. Tese (Doutorado em Zootecnia) - Universidade Estadual de Maringá, Maringá.

Sosa, D.A. (2008). Carne ovina: produção doméstica e importações. . Available at: $<$ http://www.farmpoint.com.br/carne-30-.ovina-producao-domesticaimportacoes_noticia_47320_1_2_.aspx> Accessed on: Nov. 20, 2010.

Walker, G. P., F. R. Dunshea, and P. T. Doyle. (2004). Effects of nutrition and management on the production and composition of milk fat and protein: A review. Aust. J. Agric. Res. 55:1009-1028. 